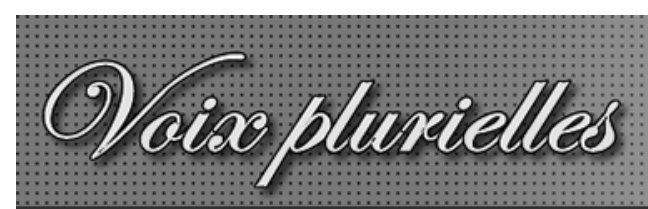

Revue de l'Association des Professeur-e-s de Français des Universités et Collèges Canadiens

\author{
Voix plurielles \\ Volume 5, Numéro 1 : mai 2008
}

Bertrand Bourgeois et Élise Lepage

\title{
Introduction : La maison et le livre
}

Citation MLA : Bourgeois, Bertrand et Élise Lepage. «La maison et le livre.» Voix plurielles 5.1 (mai 2008).

(C) Voix plurielles, revue électronique de l'APFUCC 2008. 


\title{
La maison et le livre
}

\author{
Bertrand Bourgeois, Université Western Ontario \\ Élise Lepage, Université de Colombie-Britannique
}

Mai 2008

Ce projet n'aurait pu voir le jour sans l'idée originelle et originale d'Hélène Cazes qui, au congrès 2007 de l'Association des Professeur.e.s de Français des Universités et Collèges Canadiens (APFUCC), proposa un atelier portant sur les «Espaces du livre ». Au cours de cet atelier, la maison et le livre apparurent comme des structures échangeant des rapports à géométrie variable, des espaces communicants qui s'enchâssent, se contiennent et se métaphorisent mutuellement. «Les images de la maison marchent dans les deux sens : elles sont en nous autant que nous sommes en elles », écrit Gaston Bachelard dans Poétique de l'espace. «Les images de la maison marchent dans les deux sens : elles sont dans les livres autant que les livres sont en elles », pourrait-on réécrire.

Parce qu'ils se constituent comme espaces délimités de signification, maison et livre invitent, chacun à leur façon, à interroger notre habitation du monde référentiel et livresque. Comment s'effectue cette habitation et quelle(s) forme(s) prend elle? Toute œuvre littéraire ne tente-t-elle pas, ultimement, de donner forme au monde, de l'aménager provisoirement à travers le langage, tout comme la maison instaure une forme primordiale à l'aune de laquelle penser de plus vastes ensembles? La littérature et son apparat critique, mais aussi plus largement les sciences humaines n'ont pas manqué d'interroger ces figures fondamentales de l'imaginaire littéraire. Gérard Genette a ainsi mis l'accent sur les «seuils»du texte alors que Philippe Hamon a insisté sur l'importance de la métaphore architecturale pour mieux cerner la littérature du XIX ${ }^{\mathrm{e}}$ siècle, tous deux entraînant derrière eux une partie de la critique littéraire amenée à reconsidérer le topos théorique qui veut que le texte et le récit soient liés au temps plutôt qu'à l'espace. Plusieurs métaphores récurrentes empruntées à l'architecture et au monde des bibliothèques (cathédrale, seuils, portes et fenêtres, bibliothèque labyrinthique, hôtels et lieux de passages, etc.) témoignent de ce que l'on pourrait désigner comme une « révolution spatiale » de la critique, amorcée depuis les années 1980.

Beaucoup de ces métaphores consistent à rappeler que si le récit peut être pensé, en termes aristotéliciens, comme une succession chronologique d'événements, il peut également être conceptualisé comme le parcours d'une espace circonscrit, et que la dimension profondément matérielle de la maison est aussi une caractéristique (trop souvent oubliée) du livre. Il est vrai que de tous temps, la matérialité du texte écrit a informé sa lecture. Premier temps de la réflexion, la section «Seuils » interroge l'analogie entre livre et maison à l'époque médiévale et renaissante, moments forts de l'histoire de cette analogie puisque, comme le montre Carlos F. Clamote Carreto, le livre et le tombeau ont partie liée dans une culture essentiellement orale où les livres et les demeures ultimes sont parmi les seules productions à être appelées à demeurer. L'article d'Hélène Cazes montre quant à lui à travers l'exemple de l'œuvre de Charles Estienne 
comment le parcours de lecture, d'abord métaphorisé sous forme de promenade devient peu à peu consultation érudite du livre en bibliothèque. Ce passage de la métaphore du jardin à la maison n'est pas sans influencer la forme de l'œuvre en même temps qu'il semble signifier un changement épistémologique majeur dans une Histoire où le livre devient l'instrument didactique d'un savoir objectif et la lecture une pratique réglée et méthodique...

$\mathrm{Au} \mathrm{XIX}^{\mathrm{e}}$ siècle, l'Histoire s'accélère brusquement pour les mondes respectifs des livres et de l'habitation. D'un côté, les débuts de l'industrie du livre et l'augmentation progressive du nombre de lecteurs modifient la production littéraire. De l'autre, l'urbanisation implique de repenser l'habitation (prix accessibles, préoccupation croissante pour l'hygiène, spécialisation des pièces, réduction constante de la dimension des domiciles). Ce double processus transparait dans la pièce qui constitue le croisement par excellence des figures livresques et domestiques : la bibliothèque. Jeremy Worth montre comment les bibliothèques des Rougon-Macquart informent le projet zolien de «tout dire pour tout connaître, pour tout guérir »; Bertrand Bourgeois étudie quant à lui les représentations de bibliothèques de fameux bibliophiles réels et fictifs de la fin-de-siècle (les Goncourt et le personnage de Des Esseintes) et expose comment dans La maison d'un artiste et dans À Rebours l'esthétisation «décadente » du texte s'exprime par la mise en place de la «maison-texte» livresque. Claire Le Thomas s'intéresse aux mutations que connaissent les bibliothèques particulières à la même époque et au début du $\mathrm{XX}^{\mathrm{e}}$ siècle : dès la fin du $\mathrm{XIX}^{\mathrm{e}}$ siècle apparât ainsi toute une littérature concernant les travaux manuels amateur destinés à l'entretien et à l'aménagement du logis.

Cette réflexion se poursuit dans la première moitié du $\mathrm{XX}^{\mathrm{e}}$ siècle dans les milieux de l'avantgarde artistique, mais aussi sous des plumes féminines. Comme le souligne Murielle Martin, l'avant-garde picturale en bouleversant l'espace de la toile invite à repenser les liens entre le texte et l'image à travers la figuration architecturale, ce dont témoigne en particulier l'œuvre à la fois visuelle et littéraire du peintre De Chirico où la représentation textuelle ou plastique d'espaces d'habitation est un mythe créateur récurrent. De son côté, Francesca Negro se penche sur plusieurs œuvres d'un des représentants majeurs du théâtre de l'absurde au $\mathrm{XX}^{\mathrm{e}}$ siècle, Eugène Ionesco, et montre comment sa représentation dramaturgique de l'espace domestique peut être mise en relation avec les expérimentations modernistes entreprises par Le Corbusier à la même époque.

Par ailleurs, plusieurs auteures ont écrit sur les lieux, et plus précisément les maisons dans lesquelles elles ont fait œuvre. Le sujet féminin entretient-il des liens particuliers avec ses maisons de l'écrit? Comment s'y inscrit-il et, en retour, comment les représente-t-il? Cette perspective qu'ouvre le regard féminin sur la problématique maison-livre invite également à interroger certains lieux communs : la maison traditionnellement perçue comme un espace féminin et l'écriture comme un acte viril, par opposition à la lecture qui serait une activité massivement féminine, etc. Telles sont quelques unes des interrogations auxquelles se confrontent les articles respectivement consacrés à Colette par Josette Rico, à Anaïs Nin par Maud Rauturier et à Marguerite Duras par Dominique Denes.

«Architectures du texte postmoderne »s'intéresse aux actualisations les plus récentes des liens entre livre et maison. La lecture des différents articles de cette section fait ressortir quelques dimensions particulièrement frappantes. La problématique s'inscrit plus largement dans un regard quelque peu effaré sur le monde contemporain, monde qui multiplie signes et objets, lieux et espaces mais les désémantise ou les délocalise tout autant. Maison et livre sont dorénavant 
conçus sur le mode de l'inhabitable, de l'illisible, de la multiplicité et du non-sens. Plusieurs articles s'intéressent à ces formes d'habitation passagères que sont les hôtels, les refuges, les aéroports, etc. alors que d'autres montrent à quel point l'expérience de la prison, des camps d'extermination, de l'errance forcée compromettent toute possibilité de foyer et d'écriture. L'article de Louise Lachapelle ouvre la section, car en plus de toucher à de nombreuses œuvres, il pose en s'intéressant à L'Espèce humaine d'Antelme la rupture épistémologique qui fonde l'impossibilité de prendre désormais la question du texte et de la maison pour acquise: Auschwitz et les camps de concentration. Frédéric Marteau présente trois formes de constructions autour de l'œuvre de Thomas Bernhard: contructions d'habitats, constructions de pensées et constructions verbales alors qu'à la lumière de l'ouvrage protéiforme Habit Habitat Habitus, Élise Lepage avance le concept de domestication afin de cerner la poétique des œuvres de Pierre Morency et de Pierre Nepveu. Valérie Dupuy plonge dans les méandres labyrinthiques de La Maison des feuilles de Mark Z. Danielevski, œuvre dont la structure hautement expérimentale pousse à bout les relations entre maison et livre. La fin de cette section interroge la figure de la maison par le biais paradoxal de la mobilité, de l'impossible habitation : Isabelle Dangy propose une astucieuse lecture guidée des 43 chambres d'hôtel de Suite à l'hôtel Crystal d'Olivier Rolin, tandis que Clara Domingues se penche sur la pièce Le Voyageur sur le banc de Reza Daneshvar, dont le protagoniste hante l'aéroport Charles de Gaulle à défaut de pouvoir le quitter; ce lieu d'habitation impossible le conduit à élaborer des mises en récit de sa vie de plus en plus fictives, à écrire une pseudo-biographie en mille-feuilles construisant peu à peu un refuge textuel.

En soulignant les liens que noue la problématique avec l'intertextualité, la dernière section se donne pour objectif de montrer que la maison et le livre sont tous deux des supports, des lieux de mémoire privilégiés. Une mémoire du sujet, tout autant qu'une mémoire palimpseste des textes : tel est ce à quoi s'attache l'article de Chrystelle Claude, consacré aux œuvres de Peter Ackroyd et de Pascal Quignard, ainsi que celui d'Ilaria Vitali, qui s'attache aux réécritures des Mille et une Nuits dans la littérature algérienne contemporaine.

Jusqu'où peut-on suivre Daniel Pennac lorsqu'il écrit que «l'homme construit des maisons parce qu'il est vivant, mais il écrit des livres parce qu'il se sait mortel » (Comme un roman)? La maison n'a-t-elle pas elle aussi tout à voir avec la mort (le camp, le tombeau ou le mausolée) et le livre avec la vie (j'écris de mon vivant et pour demeurer à la postérité, avec des textes et pour des textes que je veux maintenir en vie)?

Pour avoir initié ce questionnement et participé à la réalisation de ce projet, nous remercions donc chaleureusement Hélène Cazes, le rédacteur de Voix plurielles Jean Ntakirutimana, ainsi que l'ensemble des auteurs qui, chacun à leur manière, ont montré comment maison et livre se révèlent être des espaces fondamentaux de l'imaginaire littéraire, irrémédiablement ouverts l'un sur l'autre. 